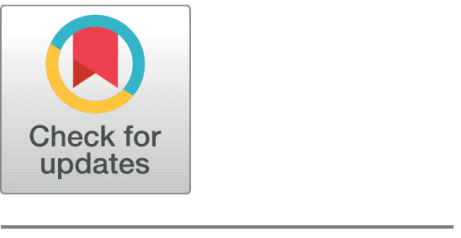

G OPEN ACCESS

Received: 28.09.2021

Accepted: 05.01.2022

Published: 31.01 .2022

Citation: V G V, K T (2022) Spatial-Spectral Feature for Extraction Technique for Hyperspectral Crop Classification. Indian Journal of Science and Technology 15(2): 81-90. https://doi. org/10.17485/IJST/v15i2.1810

* Corresponding author.

vanivg@gmail.com

Funding: None

Competing Interests: None

Copyright: $\Subset 2022$ V G \& K. This is an open access article distributed under the terms of the Creative Commons Attribution License, which permits unrestricted use, distribution, and reproduction in any medium, provided the original author and source are credited.

Published By Indian Society for Education and Environment (iSee)

ISSN

Print: 0974-6846

Electronic: 0974-5645

\section{Spatial-Spectral Feature for Extraction Technique for Hyperspectral Crop Classification}

\author{
Vani $\mathbf{G}^{1 *}$, Thippeswamy $\mathrm{K}^{2}$ \\ 1 Associate professor, Department of Computer science \& Engineering, Government \\ Engineering College, Kushalnagar, India \\ 2 Professor \& Head, Department of PG studies, VTU Regional office, Mysore, India
}

\section{Abstract}

Objectives: To present an extraction technique for the classification of the hyperspectral crop using the spatial-spectral feature. Methods: This paper presents a spatial-spectral feature extraction method employing the Image fusion technique and intrinsic feature extraction and a model for Improved Decision Boundary (IDB) using Support Vector Machine (SVM). Findings: The experiments have been conducted by using the Indian pines dataset which was extracted using the AVIRIS sensor. The dataset comprises of 16 distinctive classes such as corn, wheat, oats etc, which have used for evaluation of our model. Before the evaluation of the dataset the model has been trained using different training datasets in order to increase the accuracy and reduce misclassification. Moreover, the Spatial-Spectral Feature (SSF) model aided in distinguishing between crop intrinsic features and shadow element under dynamic environment condition. Our model attained 99.54\%, 99.4\%, 99.25\% and $9.8 \mathrm{sec}$ for OA accuracy, AA accuracy, Kappa and Time respectively. Furthermore, the overall accuracy of the model for the Support Vector Machine-3-dimensional discrete wavelet transform (SVM3DDWT), Convolutional Neural Network and Spatial-Spectral Feature Extraction Technique showed result of $94.28 \%, 96.12 \%$ and $99.4 \%$ respectively. Other existing models showed a low accuracy for the same dataset. Further, for addressing class imbalance issues this work modelled an improved decision boundary model for SVM by considering soft-margin rather than hard margin. The SSF-IDBSVM model achieves much better accuracies with less misclassification in comparison with recent deep learning-based HSI classification model. Novelty: Recently, several feature extraction and deep learning-based crop classification model have been modelled. However, existing crop classification fails to distinguish crop intrinsic feature concerning shadow feature; further, consider hard decision boundary; as a result, high misclassification is induced for smaller class size. Hence, this study provides an extraction feature which provides the classification of the crop in less time with higher classification and less misclassification. 
Keywords: Artificial Intelligence; Datamining; Crop Classification; Feature Extraction; Feature Selection; Hyper Spectral Information; Machin Learning Technique

\section{Introduction}

The crop classification model is one of the principal components of agriculture crop checking by using hyperspectral imaging acquired through satellites. Harvest planning through hyperspectral imaging arrangement help in settling on the different dynamic in farming climate, for example, yield determining, crop region evaluation, etc. ${ }^{(1,2)}$. Precision crop planning is vital and sways crop recognizable proof applications. In any case, any obstacles that exist in crop identification utilizing hyperspectral imaging should be met ${ }^{(3)}$. To start with, the conceivable high measurement size of hyperspectral imaging information is as yet an open issue. Second, hyperspectral imaging information takes after high likenesses of surfaces, spectral signatures marks, and shapes among various yields. Ultimately, the existence of mixed pixels in hyperspectral imaging altogether sways the correctness of the existing hyperspectral imaging crop arrangement model.

With the development of hyperspectral imaging systems, the spatial resolutions have also been improved. Along these lines, examining hyperspectral imaging is troublesome. Moreover, by consideration of the personality of the hyperspectral imaging there exists a higher connection between the adjoining spectral band set and pixels ${ }^{(4-6)}$. At that point, due to the large dimensions of the image in the hyperspectral image, there are more chances of expansion in space-time, and overhead computation ${ }^{(7)}$. Subsequently, decreasing the measurement size of the repetitive components in HSI is fundamentally essential for handling hyperspectral pictures. Band Selection ${ }^{(8-10)}$ is one of the proficient measurements for reducing the dimension spectrally by eliminating excess features. The main aim of using the band selection is to pick the most productive subset of a band which is made out of data inside unique groups. On contrary, the feature extraction models ${ }^{(11)}$ lessens measurement size as per intricate feature change. Consequently, feature selection techniques are clearer and can be utilized for useful reasons. The component determination technique can pick groups just inside hyperspectral pictures. On the other hand, the element extractor can utilize the hyperspectral image band set to produce an improved separating highlight set. The Laplacian discriminant examination of hyperspectral image ${ }^{(11)}$, introduced joint feature extraction and feature selection strategies for hyperspectral image classification. Like ${ }^{(11)}$ their work centers around building up the dimensionality decrease strategy by mining the semantically helpful list of capabilities from the raw HSI.

Various sorts of HSI dimensional decrease strategies that incorporate both unsupervised and supervised methods have been introduced in late time. This model centers on supervised philosophies, for example, a linear discriminant investigation ${ }^{(12)}$, and $\operatorname{ICA}^{(13)}$, and PCA ${ }^{(14)}$. From the examination, it is seen that foremost part investigation procedures extract much better components when contrasted and different approaches. The PCA highlight extraction procedure can ensure that further related elements of hyperspectral imagery can be reserved with the negligible size of valuable head parts. On contrary, ICA-based procedures can ensure that the changed segments are free just about as much as possible. However, independent component analysis-based procedures induce high computational overhead because of their unpredictable calculation. Further, the independent component analysis systems don't consider spatial setting data for removing highlights as they treat and interact with every pixel freely. The larger part of the existing hyperspectral imagery measurement decrease procedure can't straightforwardly use spatial data of hyperspectral pictures and the highlight extraction technique utilizes just phantom data of each pixel which 
is expressed to be productive for upgrading the characterization correctness's $(15,16)$. The earth's climate is made out of various kinds of crops like soil, wheat, corn, and so forth hyperspectral image characterization strategy targets recognizing this significant portrayal of harvests. Consequently, it is important to mine significant spatial capabilities. Thusly, for removing significant spatial data from the HSI made out of blended pixel, it is imperative to catch intrinsic properties of actual attributes of various crops. Along these lines by separating spatial data from the neighbor crops can help in expanding the precision of separating various harvests present in earth climate. The intrinsic HSI is composed of shading components and object intrinsic features. Extricating these parts assumes a vital part in crop identification jobs and simultaneously it is trying to remove these segments from a single HSI. This is because intrinsic capabilities depend on crops highlights of the earth and differ regarding environmental and climatic conditions. Accordingly, for separating more significant spatial elements of various yields, this work presents an effective intrinsic feature extraction technique namely Spatial-Spectral Feature (SSF) that keeps a spatially and spectrally significant portrayal of various harvests. Then, for decreasing the hyperspectral band measurement size that is productive against mixed pixel and noisy conditions, the image fusion strategy presented in ${ }^{(17)}$ is used. Later these features extracted are trained using SVM. However, achieve very poor classification accuracy especially for the crop with less feature; To address class imbalance issues number of Deep learning-based classification models has been emphasized in recent times $^{(18-22)}$. However, most of the existing deep learning-based HSI object classification model ${ }^{(18,23-28)}$ achieves very good classification accuracies but induces more computation overhead; Further, this model requires a high number of features for training model and classification model are designed considering hard decision boundaries; To overcome these issues, this work employs improved decision boundary (IDB) by considering soft-margin for SVM ${ }^{(29,30)}$. The SSF trained using IDBSVM aided in overcoming class imbalance issues, reducing misclassification, and improving classification accuracies.

The rest of the paper is organized as follows. In section II spatial-spectral feature and improved decision boundary support vector machine-based hyperspectral crop classification is presented. Section III discusses the outcome achieved using SSFIDBSVM and other existing crop classification models. The last section's significance of work is discussed and future research direction put forth.

\section{Hyperspectral Crop Classification using Spatial-spectral feature and Improved Decision Boundary Support Vector Machine}

This section presents hyperspectral crop classification using improved feature extraction of spatial-spectral features and also presents enhanced decision boundaries that can work well under a mixed cropping environment. The working of SSF-IDBSVM is shown in Figure 1.

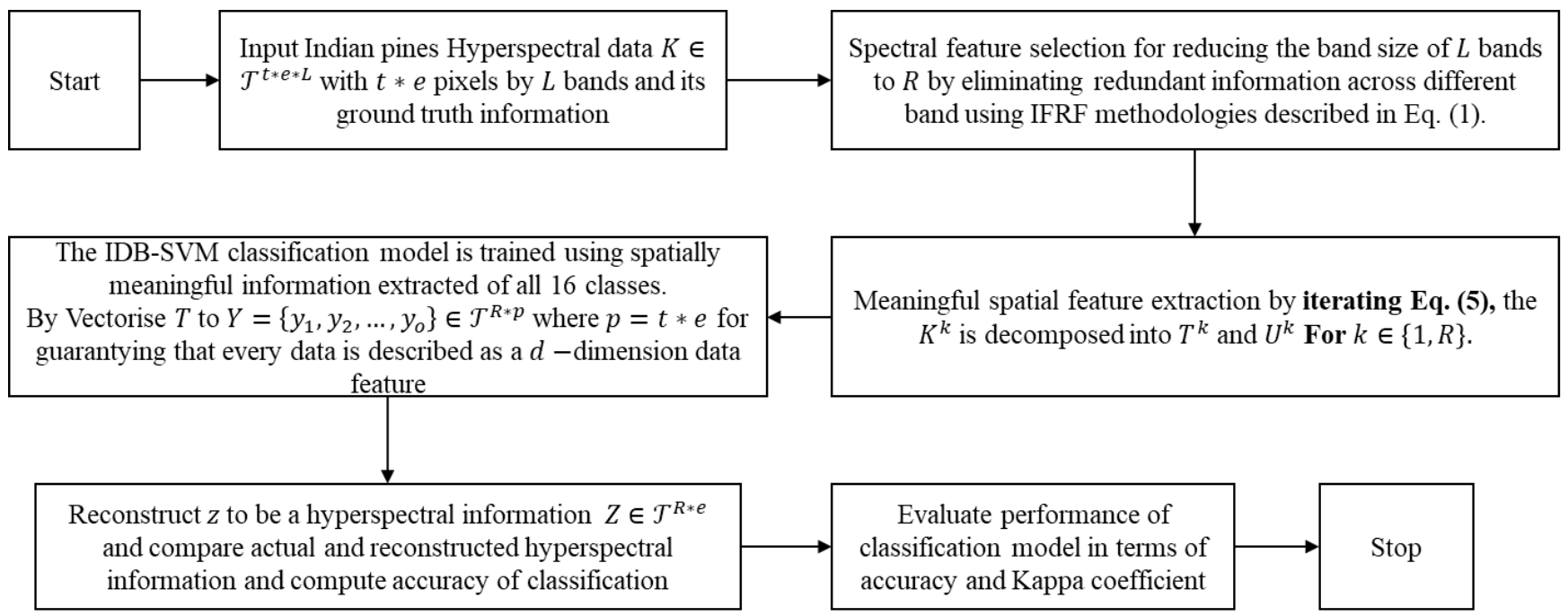

Fig 1. The architecture of Spatial-Spectral feature using IDBSVM based Hyperspectral Crop Classification 


\subsection{Spatial-spectral feature extraction for crop classification:}

This section presents feature selection methodologies for agriculture crop classification. This model is designed to bring a tradeoff between reducing feature size and retaining useful information; that is the work reduces the $L$ bands to $R$ bands through segmentation process applying fusion methodologies. This work considers fusion methodologies ${ }^{(8)}$ rather than PCA because of high spectral information retain efficiency as described as follows

$$
L^{m}=\frac{\sum_{n=(m-1) 0+1}^{m o} k^{n}}{o}, m=\left(\frac{L}{R}\right)
$$

where $m$ defines the dimension of real spectral band indices information which can be obtained using the following equation

$$
m=\left(\frac{L}{R}\right\rfloor
$$

and $n$ define the $n^{\text {th }}$ dimension of real spectral band indices information, $K^{n}$ defines repeated feature spectral data in every band, and $o$ defines the sub-group band size of each band. The above equation is used for removing repeated features within each subgroup.

Generally, multiple crops have been grown in an agricultural environment. The spectral information is captured using a satellite. Existing methodologies randomly extract these features and train the machine learning model and then perform crop classification. However, each crop has its absorption and reflectance properties concerning varying climatic conditions. Therefore, it is important to extract crop intrinsic properties to obtain better features aiding superior classification performance. Let the intensity value of hyperspectral data be $K \in T^{t * e}$ where $U \in T^{t * e}$ and $T \in T^{t * e}$ describes shading features and intrinsic features, respectively; Thus, hyperspectral data of respective pixel $r$ intrinsic feature can be represented as follow

$$
K_{r}=T_{r} U_{r}
$$

where $r$ describe its pixel's locations. The shading component parameter $U_{r}$ is computed as follows

$$
U_{r}=\frac{1}{K_{r}}
$$

and the reflectance properties $T_{r}$ is computed using the following equation

$$
T_{r}=\sum_{s \in P(r)} c_{r s} T_{s},
$$

where $r$ and $s$ describes pixel matrix positions, $P(r)$ describes adjacent pixel $r$ and $c_{r s}$ depicts features of pairwise similarity matrix between $K_{r}$ and $K_{s}$. Thus, using Eq. (1) to (5) we establish reflectance value for each crop by mitigating shading concerning semantic features. Thus, we will have the spatial-spectrally useful feature by eliminating useless spatial information of shading elements across different bands. Then, the features of each crop are trained using an improved decision boundary support vector machine for multiclass crop classification.

\subsection{Agriculture crop classification using improved decision boundary support vector machine:}

To classify the optimal hyperspectral feature set, this model utilizes the pixel-support-vector machine classification technique. SVM is usually used to solve regression, classification, and detection problems. The SVM builds a hyperplane or a set of hyperplanes in the high dimensional space. Hence, from ${ }^{(14)}$ we can know the structure of SVM. Therefore, the hyperplane linear model of SVM can be defined as

$$
y=w^{T} \phi(X)+b
$$

where $\phi(X)$ is transformed feature space. The margin is defined as the smallest distance from the decision hyperplane to the closest point from the dataset. In the SVM problem, we are trying to construct the decision boundary hyperplane to maximize the margin with the dataset. For each data point, $t_{i}$ is the target label, where $t \in(1,-1\}$. In our case, the problem is not linearly separable, we will use soft-margin SVM and introduce slack variable $\varphi_{i} \geq 0 . \varphi$ allows the misclassification of the outline. When $\varphi_{i}>1$, the data point is misclassified. At the same time, we should have inequality constraint as follows:

$$
t_{i}\left(W_{i}^{T} \phi_{i}(X)+b\right) \geq 1-\varphi_{i}
$$


To find the maximum margin solution, we solve the following problem:

With the simplification of the problem and the soft-margin slack variable, the SVM problem eventually becomes:

In Equation (9) the variable $C$ is utilized to regularize the parameter which can control the trade-off among the tolerance and margin of misclassification. Moreover, as the dataset is non-linearly separable, the kernel trick which is used to transform the data to a high linear dimension like the Gaussian Kernel can be used. As the SVM problem is a convex optimized problem, a globally optimized solution can be obtained from the model. With the optimal decision boundary, we can use it to classify our dataset into different labels. However, through the experimental study we have noticed that when a high noise and a mixedcropping environment is applied, a large number of false positives are obtained. For addressing an improved decision boundary is introduced in below equation

where $\delta_{1}$ and $\delta_{2}$ represent a variable that is used for bringing ideal crop classification outcomes, $\bar{\gamma}$ and $\widehat{\gamma}$ represent decision boundaries difference and decision boundaries mean, respectively. These boundaries are computed using the following equations

$$
\begin{gathered}
\widehat{\gamma}=\frac{1}{n^{2}} \sum_{j=1}^{n} \sum_{k=1}^{n}\left(z_{j} x^{U} \alpha\left(y_{j}\right)-z_{j} x^{U} \alpha\left(y_{j}\right)\right]^{2} \\
\bar{\gamma}=\frac{1}{n} \sum_{j=1}^{n} z_{j} x^{U} \alpha\left(y_{j}\right)=\frac{1}{n}(Y z)^{U} x,
\end{gathered}
$$

The proposed spatial-spectral feature trained with improved decision boundary-SVM crop classification achieves much better classification accuracy when compared with existing crop classification methodologies which are by experimentation shown in the next section.

\section{Results and Discussions}

In this section performance evaluation of the proposed SSF-IDBSVM model is carried out, performance evaluation is carried out by comparing the proposed HSI-based crop classification model with the existing crop classification model ${ }^{(23)}$; the further model is evaluated on the data which is gathered from the AVIRIS sensor ${ }^{(31)}$. This dataset comprises 224 spectral reflectance bands along with $0.42 .5^{\star} 10^{-6}$ meters wavelength and also it comprises class variables from vegetation, forest, and agriculture. Dataset has 16 distinctive classes used for the evaluation; further detail of the dataset is given in Table 1. Indian Pine hyperspectral data is captured through the AVIRIS sensor; dataset holds 10249 samples. Comparative analysis of proposed crop classification and various existing crop classification methods is performed. Moreover, the evaluation is carried out on basis of average accuracy and overall accuracy ${ }^{(17)}$; overall accuracy shows the absolute classification over the complete test feature an average accuracy shows the individual class average; moreover, in the case of all the metrics higher value indicates the superiority of classification approach.

\subsection{Indian Pines Dataset}

The Indian Pines Dataset is a standard Hyperspectral Image Segmentation dataset. The input data consists of hyperspectral bands over a single landscape in Indiana, US, (Indian Pines data set) with $145 \times 145$ pixels. For each pixel, the data set contains 220 spectral reflectance bands which represent different portions of the electromagnetic spectrum in the wavelength range $0.4-2.5 \cdot 10-6$. The Indian Pines scene contains two-thirds agriculture, and one-third forest or other natural perennial vegetation. There are two major dual lane highways, a rail line, as well as some low-density housing, other built structures, and smaller roads. Since the scene is taken in June some of the crops present, corn, soybeans, are in early stages of growth with less than $5 \%$ coverage. The ground truth available is designated into sixteen classes and is not all mutually exclusive. Indian Pines data are available through Pursue's univeristy MultiSpec site. 
Table 1. The total number of features present in each crop of Indian Pines Hyperspectral data.

\begin{tabular}{lll}
\hline Number & Classes & Total Samples \\
\hline $\mathbf{1}$ & Alfalfa & 46 \\
$\mathbf{2}$ & Corn notill & 1428 \\
$\mathbf{3}$ & Corn mintill & 830 \\
$\mathbf{4}$ & Corn & 237 \\
$\mathbf{5}$ & Grass pasture & 483 \\
$\mathbf{6}$ & Grass trees & 730 \\
$\mathbf{7}$ & Grass pasture moved & 28 \\
$\mathbf{8}$ & Hay windrowed & 478 \\
$\mathbf{9}$ & Oats & 20 \\
$\mathbf{1 0}$ & Soybean notill & 972 \\
$\mathbf{1 1}$ & Soybean mintill & 2455 \\
$\mathbf{1 2}$ & Soybean clean & 593 \\
$\mathbf{1 3}$ & wheat & 205 \\
$\mathbf{1 4}$ & woods & 1265 \\
$\mathbf{1 5}$ & Buildings Grass Trees Drives & 386 \\
$\mathbf{1 6}$ & Stone Steel Towers & 93 \\
\hline
\end{tabular}

\subsection{Classification performance achieved using Indian Pines dataset:}

Here the performance of SSF-IDBSVM-based hyperspectral crop classification performance outcome achieved is compared with standard SVM, multiscale joint collaborative representation with a locally adaptive dictionary (MLJCRC) ${ }^{(32)}$, and $\mathrm{CNN}-\mathrm{AL}_{-\mathrm{MNF}}{ }^{(23)}$ based hyperspectral crop classification. Here we have considered 5\% data for training and accuracies and misclassification i.e., Kappa coefficient is metrics considered for validating hyperspectral crop classification model. The remaining $95 \%$ of data is used for testing the hyperspectral crop classification model. The result attained by using different crop classification models is sown in Figure 2 and Table 2. From the overall result attained it can be seen the SSF-IDBSVM achieves much better classification accuracies with less misclassification when compared with MLJCRC and CNN-AL-MNF-based crop classification model. Further, we can notice using IDBSVM significantly reduces computation overhead in comparison with deep learning-based hyperspectral crop classification.

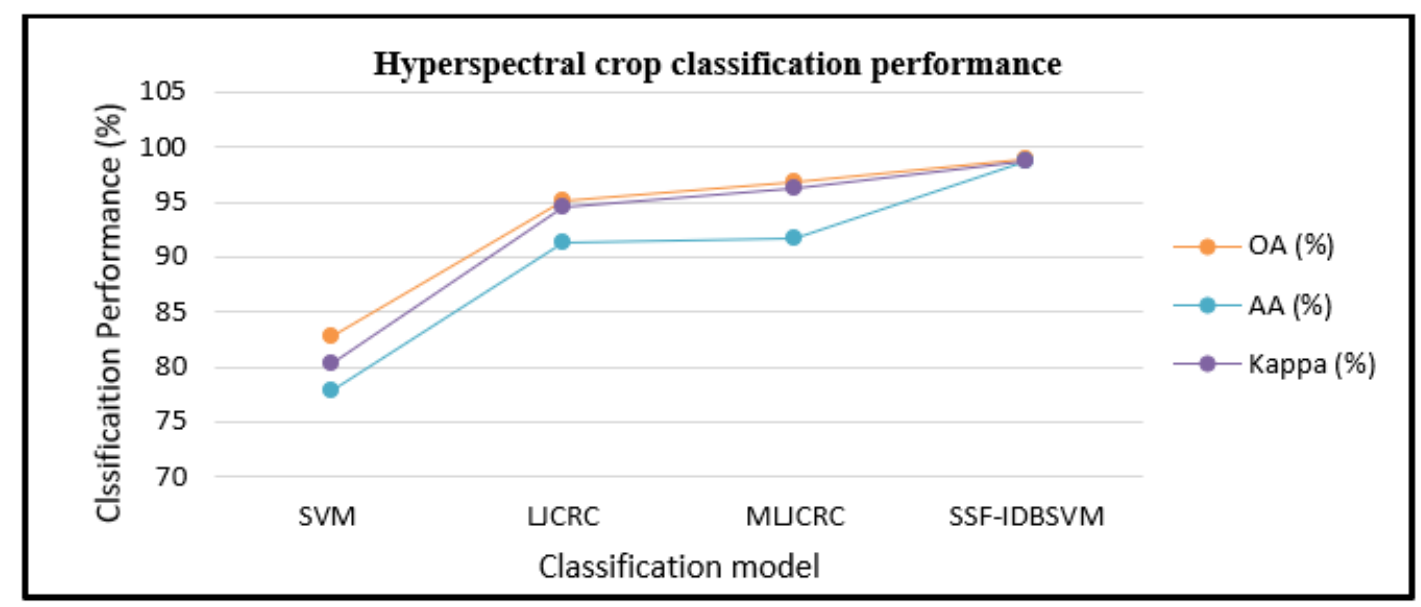

Fig 2. The classification outcome was attained using SSF-IDBSVM and the existing hyperspectraldata-based crop classification model. 
Table 2. The classification outcome attained by the proposed HSI classification method over thestate-of-art HSI classification method

\begin{tabular}{|c|c|c|c|c|c|c|}
\hline Class name & Train & $\mathbf{S V M}^{(30)}$ & LJCRC $^{(32)}$ & MLJCRC ${ }^{(32)}$ & CNN-AL-MNF ${ }^{(23)}$ & SSF-HSI \\
\hline Alfalfa & 6 & 70.37 & 87.96 & 94.44 & 84.17 & 100 \\
\hline Corn notill & 144 & 68.87 & 91.35 & 93.27 & 91 & 100 \\
\hline Corn mintill & 84 & 73.82 & 87.35 & 94.24 & 83.64 & 99.74 \\
\hline Corn & 24 & 57.98 & 93.16 & 99.36 & 87.01 & 100 \\
\hline Grass pasture & 50 & 92.42 & 96.38 & 96.53 & 91.57 & 99.81 \\
\hline Grass trees & 75 & 95.94 & 99.80 & 99.87 & 95.18 & 99.91 \\
\hline Grass pasture moved & 3 & 67.95 & 73.08 & 76.35 & 89.13 & 100 \\
\hline Hay windrowed & 49 & 98.09 & 100 & 100 & 98.93 & 98.99 \\
\hline Oats & 2 & 50.00 & 30.00 & 12.50 & 16.08 & 98.11 \\
\hline Soybean notill & 97 & 79.82 & 89.82 & 92.92 & 90.68 & 97.02 \\
\hline Soybean mintill & 247 & 85.63 & 98.18 & 98.91 & 94.7 & 98.41 \\
\hline Soybean clean & 62 & 70.20 & 97.07 & 98.25 & 91.51 & 99.94 \\
\hline wheat & 22 & 97.27 & 99.53 & 100 & 99.25 & 100 \\
\hline woods & 130 & 96.86 & 97.68 & 98.88 & 95.73 & 100 \\
\hline Buildings Grass Trees Drives & 38 & 49.12 & 88.55 & 90.86 & 83.996 & 98.56 \\
\hline Stone Steel Towers & 10 & 81.40 & 98.27 & 98.35 & 92.08 & 100 \\
\hline OA (\%) & & 82.79 & 94.90 & 96.8 & 86.54 & 99.54 \\
\hline $\mathrm{AA}(\%)$ & & 80.33 & 91.22 & 91.63 & 92.26 & 99.4 \\
\hline Kappa (\%) & & 79.44 & 94.5 & 96.34 & 0 & 99.25 \\
\hline Time (s) & & 23.60 & 58.44 & 545.81 & 5913.78 & 9.8 \\
\hline
\end{tabular}

\subsection{Effect of training varying training sample:}

Here experiment is conducted to study the effect of training sample size affecting classification performance. Here the training sample size is changed from $5 \%$ to $20 \%$ and classification outcome achieved using different crop classification models such as SSF-IDBSVM, MLJCRC ${ }^{(32)}$, CNN-MBF ${ }^{(20)}$, BS-Net-Conv ${ }^{(22)}$, and CNN-AL-MNF ${ }^{(23)}$ is measured as shown in Figure 3. From the overall result achieved we can see the proposed SSF-IDBSVM-based crop classification model achieves much better accuracies with less misclassification in comparison with existing crop classification models.

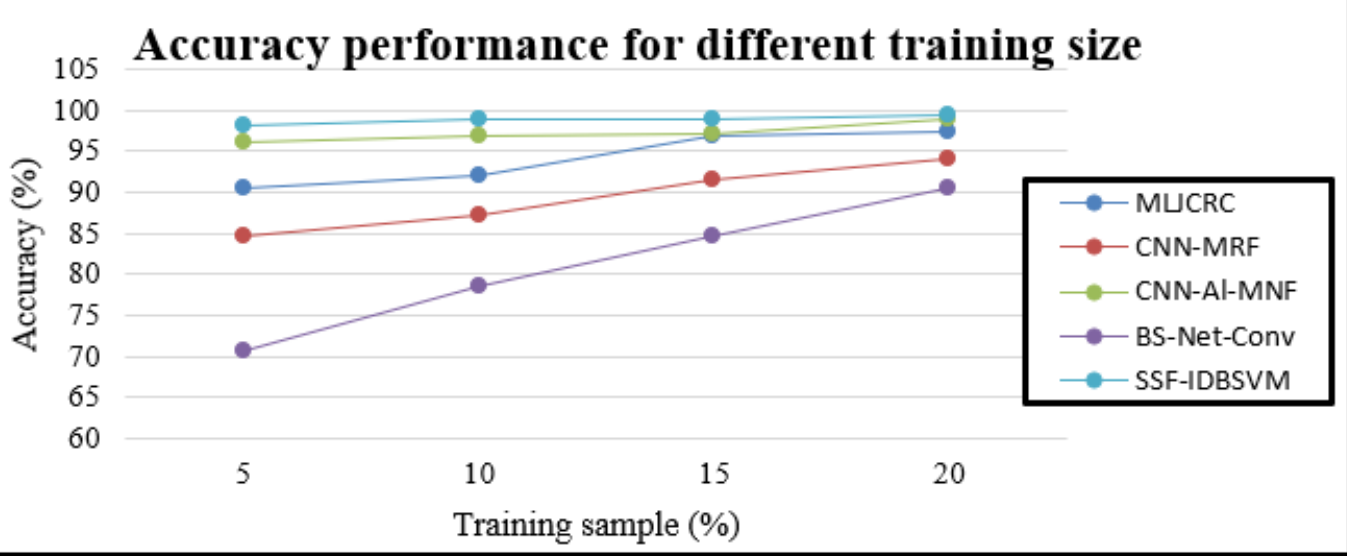

Fig 3. Effect of training sample size on classification outcome using SSF-IDBSVM and other existing crop classification models 


\subsection{Effect of spectral-spatial feature:}

Here we studied the importance of using the spatial-spectral feature in training IDBSVM. The graphical representation of classification outcomes achieved using different models such as SVM-3DDWT-GC, CNN-AL-MNF, and SSF-IDBSVM is shown in Figure 4 From Figure 4 we can see the SSF-IDBSVM outperforms other existing models. In Figure 5 the effect of using SSF is studied. From the result achieved it can be seen that using SSF significantly aided in improving classification accuracies. Thus, it proves the SSF aided in distinguishing crop intrinsic features with respect to shadow component; further, employing improved decision boundary aided in addressing class imbalance issues and achieving higher accuracies for classes with small feature sizes.

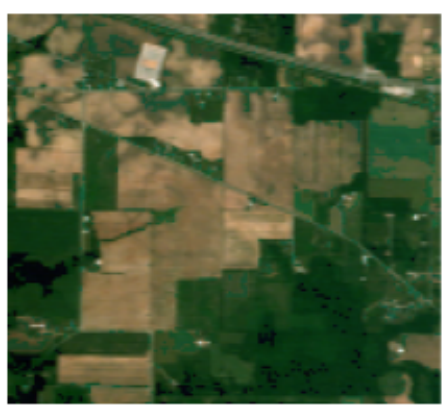

Original data

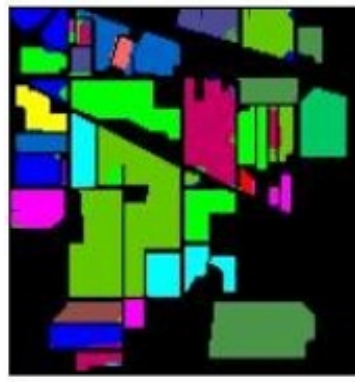

SVM-3DDWT-GC $(94.28 \%)$

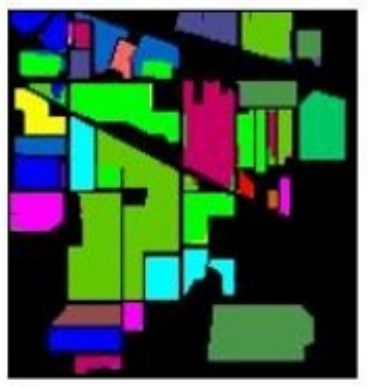

CNN-AL-MNF

$(96.12 \%)$

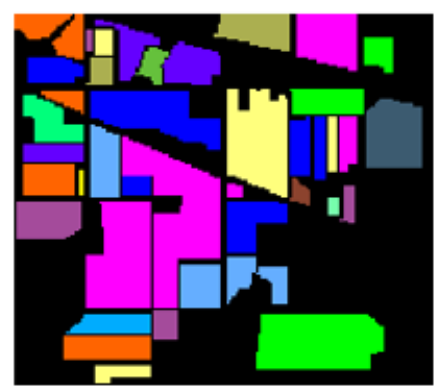

Ground truth

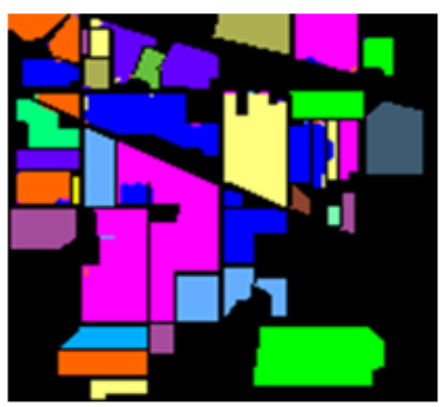

SSF-IDBSVM $(99.4 \%)$

Fig 4. Graphical representation of classification outcome obtained by all methods on the Indian Pines dataset

\section{Result and discussion}

The SSF-IDBSVM-based crop classification model attains very good results in comparison with the existing model. The existing model used a deep learning model ${ }^{(23)}$ for extracting spatial feature across different bands; however, they require high training samples and fails to obtain good feature across spectrally. On the other, the proposed model is efficiently extracted good spatial and spectral features. Similarly, the existing model ${ }^{(23,32)}$, fails to distinguish between crop features and shadow features; thus, resulting in degradation of classification accuracy. However, the proposed model uses a pairwise matrix to distinguish between shadow elements with crop features. Further, the existing model deep learning model ${ }^{(29,30)}$, uses SVM for building decision boundary; however, these model considers hard decision boundary. As a result, when there no enough training data higher is classification occurs. In addressing the proposed SSS-IDBSVM introduces a soft margin-based decision boundary aiding in the reduction of misclassification. All these novelties introduced attributed enhancement achieving better performance. 


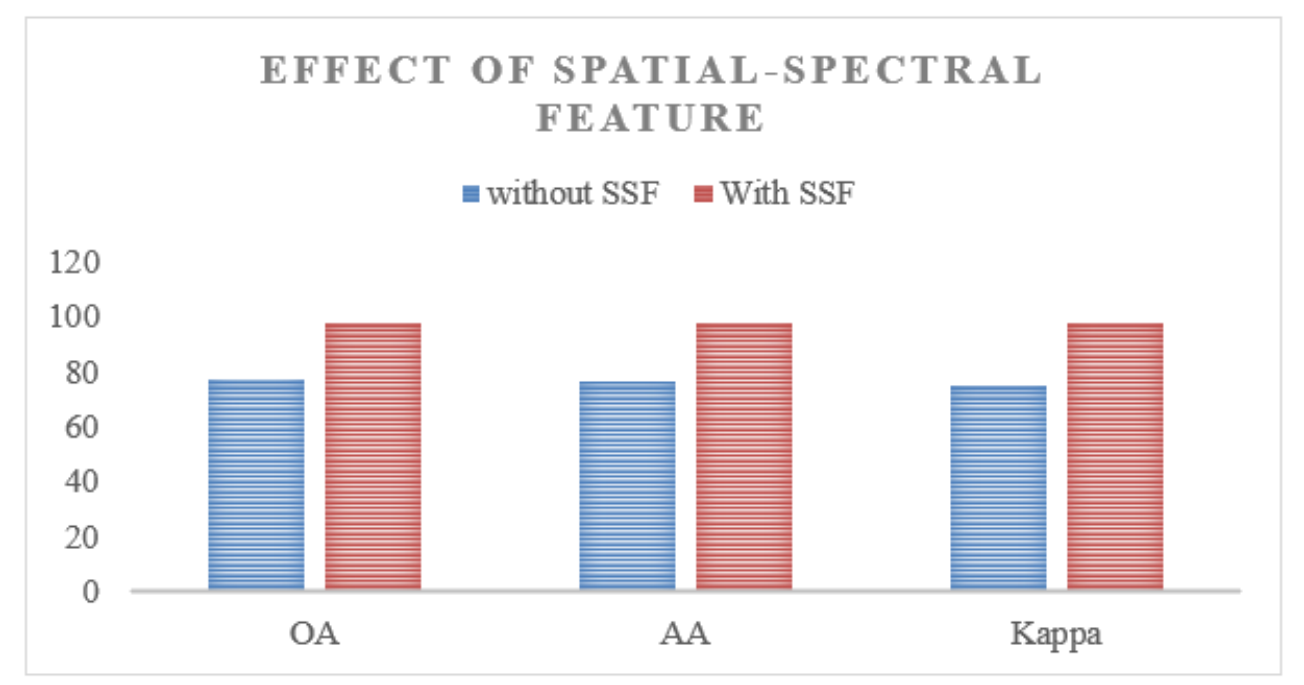

Fig 5. Graphical representation of classification outcome obtained by all methods on the Indian Pines dataset

\section{Conclusion}

This study provides a spatial-spectral feature extraction method employing the image fusion and intrinsic feature extraction mechanism. As the color of the crops keeps changing and also the climatic conditions change there are few chances that a given model may not be able to classify the given class of crops correctly which may lead to wrong results. Our Spatial-Spectral Feature (SSF) model aided in distinguishing between crop intrinsic features and shadow elements under dynamic environmental conditions. Further, for addressing class imbalance issues this work modeled an improved decision boundary model for SVM by considering soft-margin rather than hard margin. The SSF-IDBSVM model is tested using the Indian pines dataset. The metric used for validating the model is average accuracy, overall accuracy, and Kappa coefficient. Here we validated the model considering varied training sizes and also experiment is conducted to study the effect of using SSF. The overall accuracy of the models for the Support Vector Machine-3-dimensional discrete wavelet transform (SVM-3DDWT), Convolutional Neural Network and Spatial-Spectral Feature Extraction Technique showed result of 94.28\%, 96.12\% and 99.4\% respectively. In each case study, the SSF-IDBSVM model achieves significant performance in comparison with the recent HSI classification model. From the overall result attained we can see the SSF-IDBSVM outperforms other recent HSI classification models in terms of accuracy, overall accuracy, and Kappa coefficient. Further, the SSF-HIS significantly reduces computation overhead in comparison with deep learning-based HSI classification models. Furthermore, the future studies can use the different dataset to classify the different classes of crops and attain higher accuracy.

\section{References}

1) Athani SS, Tejeshwar CH. Support Vector Machine-Based Classification Scheme of Maize Crop. 2017 IEEE 7th International Advance Computing Conference (IACC). 2017;p. 84-88. doi:10.1109/IACC.2017.0032.

2) Murmu S, Biswas S. Application of Fuzzy Logic and Neural Network in Crop Classification: A Review. Aquatic Procedia. 2015;4:1203-1210. Available from: https://dx.doi.org/10.1016/j.aqpro.2015.02.153. doi:10.1016/j.aqpro.2015.02.153.

3) Tatsumi K, Yamashiki Y, Torres MAC, Taipe CLR. Crop classification of upland fields using Random forest of time-series Landsat 7 ETM+ data. Computers and Electronics in Agriculture. 2015;115:171-179. Available from: https://dx.doi.org/10.1016/j.compag.2015.05.001.

4) Gong M, Zhang M, Yuan Y. Unsupervised Band Selection Based on Evolutionary Multiobjective Optimization for Hyperspectral Images. IEEE Transactions on Geoscience and Remote Sensing. 2016;54(1):544-557. Available from: https://dx.doi.org/10.1109/tgrs.2015.2461653.

5) Sun W, Du Q. Graph-Regularized Fast and Robust Principal Component Analysis for Hyperspectral Band Selection. IEEE Transactions on Geoscience and Remote Sensing. 2018;56(6):3185-3195. Available from: https://dx.doi.org/10.1109/tgrs.2018.2794443.

6) Zhai H, Zhang H, Zhang L, Li P. Laplacian-Regularized Low-Rank Subspace Clustering for Hyperspectral Image Band Selection. IEEE Transactions on Geoscience and Remote Sensing. 2019;57(3):1723-1740. Available from: https://dx.doi.org/10.1109/tgrs.2018.2868796.

7) Eeti LN, Buddhiraju KM. Classification of Hyperspectral Remote Sensing Images by an Ensemble of Support Vector Machines Under Imbalanced Data. IGARSS 2018 - 2018 IEEE International Geoscience and Remote Sensing Symposium. IGARSS 2018 - 2018 IEEE International Geoscience and Remote Sensing Symposium. 2018;p. 2659-2661. doi:10.1109/IGARSS.2018.8519564.

8) Zhang M, Gong M, Chan Y. Hyperspectral band selection based on multi-objective optimization with high information and low redundancy. Applied Soft Computing. 2018;70:604-621. Available from: https://dx.doi.org/10.1016/j.asoc.2018.06.009. 
9) Hu P, Liu X, Cai Y, Cai Z. Band Selection of Hyperspectral Images Using Multiobjective Optimization-Based Sparse Self-Representation. IEEE Geoscience and Remote Sensing Letters. 2019;16(3):452-456. Available from: https://dx.doi.org/10.1109/lgrs.2018.2872540.

10) Wang Q, Zhang F, Li X. Optimal Clustering Framework for Hyperspectral Band Selection. IEEE Transactions on Geoscience and Remote Sensing. 2018;56(10):1-13. Available from: https://dx.doi.org/10.1109/tgrs.2018.2828161.

11) Jiang X, Song X, Zhang Y, Jiang J, Gao J, Cai Z. Laplacian Regularized Spatial-Aware Collaborative Graph for Discriminant Analysis of Hyperspectral Imagery. Remote Sensing. 2018;11(1):29-29. Available from: https://dx.doi.org/10.3390/rs11010029.

12) Huang Y, Sun Z. Semi-supervised Locality Preserving Discriminant Analysis for hyperspectral classification. 2016 9th International Congress on Image and Signal Processing, BioMedical Engineering and Informatics (CISP-BMEI). 2016;p. 151-156. doi:10.1109/CISP-BMEI.2016.7852699.

13) Jayaprakash C, Damodaran BB, V S, Soman KP. Dimensionality Reduction of Hyperspectral Images for Classification using Randomized Independent Component Analysis. 2018 5th International Conference on Signal Processing and Integrated Networks (SPIN). 2018. doi:10.1109/SPIN.2018.8474266.

14) Islam MR, Ahmed B, Hossain MA. Feature Reduction Based on Segmented Principal Component Analysis for Hyperspectral Images Classification. 2019 International Conference on Electrical, Computer and Communication Engineering (ECCE). 2019. doi:10.1109/ECACE.2019.8679394.

15) Wang L, Zhang J, Liu P, Choo KKR, Huang F. Spectral-spatial multi-feature-based deep learning for hyperspectral remote sensing image classification. Soft Computing. 2017;21(1):213-221. Available from: https://dx.doi.org/10.1007/s00500-016-2246-3.

16) Jiang J, Chen C, Yu Y, Jiang X, Ma J. Spatial-Aware Collaborative Representation for Hyperspectral Remote Sensing Image Classification. IEEE Geoscience and Remote Sensing Letters. 2017;14(3):404-408. Available from: https://dx.doi.org/10.1109/lgrs.2016.2645708.

17) Ye Z, Tan L, Bai L. Hyperspectral image classification based on spectral-spatial feature extraction. 2017 International Workshop on Remote Sensing with Intelligent Processing (RSIP). 2017;17(6):1042-1046. doi:10.1109/RSIP.2017.7958808.

18) Liang Y, Zhao X, Guo AJX, Zhu F. Hyperspectral Image Classification With Deep Metric Learning and Conditional Random Field. IEEE Geoscience and Remote Sensing Letters. 2020;17(6):1042-1046. Available from: https://dx.doi.org/10.1109/lgrs.2019.2939356.

19) Santara A, Mani K, Hatwar P, Singh A, Garg A, Padia K, et al. BASS Net: Band-Adaptive Spectral-Spatial Feature Learning Neural Network for Hyperspectral Image Classification. IEEE Transactions on Geoscience and Remote Sensing. 2017;55(9):5293-5301. Available from: https://dx.doi.org/ 10.1109/tgrs.2017.2705073. doi:10.1109/tgrs.2017.2705073.

20) Cao X, Zhou F, Xu L, Meng D, Xu Z, Paisley J. Hyperspectral Image Classification With Markov Random Fields and a Convolutional Neural Network. IEEE Transactions on Image Processing. 2018;27(5):2354-2367. Available from: https://dx.doi.org/10.1109/tip.2018.2799324.

21) Haowen L. Shorten Spatial-spectral RNN with Parallel-GRU for Hyperspectral Image Classification. Arvix. 2018. Available from: https://arxiv.org/pdf/ 1810.12563.pdf.

22) Cai Y, Liu X, Cai Z. BS-Nets: An End-to-End Framework for Band Selection of Hyperspectral Image. IEEE Transactions on Geoscience and Remote Sensing. 2020;58(3):1969-1984. Available from: https://dx.doi.org/10.1109/tgrs.2019.2951433.

23) Cao X, Yao J, Xu Z, Meng D. Hyperspectral Image Classification With Convolutional Neural Network and Active Learning. IEEE Transactions on Geoscience and Remote Sensing. 2020;58(7):4604-4616. Available from: https://dx.doi.org/10.1109/tgrs.2020.2964627.

24) Lin L, Chen C, Xu T. Spatial-spectral hyperspectral image classification based on information measurement and CNN. EURASIP Journal on Wireless Communications and Networking. 2020;2020(1). Available from: https://dx.doi.org/10.1186/s13638-020-01666-9.

25) Alotaibi B, Alotaibi M. A Hybrid Deep ResNet and Inception Model for Hyperspectral Image Classification. PFG - Journal of Photogrammetry, Remote Sensing and Geoinformation Science. 2020;88(6):463-476. Available from: https://dx.doi.org/10.1007/s41064-020-00124-x.

26) Ye M, Ji C, Chen H, Lei L, Lu H, Qian Y. Residual deep PCA-based feature extraction for hyperspectral image classification. Neural Computing and Applications. 2020;32(18):14287-14300. Available from: https://dx.doi.org/10.1007/s00521-019-04503-3.

27) Yu C, Zhao M, Song M, Wang Y, Li F, Han R, et al. Hyperspectral Image Classification Method Based on CNN Architecture Embedding With Hashing Semantic Feature. IEEE Journal of Selected Topics in Applied Earth Observations and Remote Sensing. 2019;12(6):1866-1881. Available from: https://dx.doi.org/10.1109/jstars.2019.2911987.

28) Roy SK, Krishna G, Dubey SR, Chaudhuri BB. HybridSN: Exploring 3-D-2-D CNN Feature Hierarchy for Hyperspectral Image Classification. IEEE Geoscience and Remote Sensing Letters. 2020;17(2):277-281. Available from: https://dx.doi.org/10.1109/lgrs.2019.2918719.

29) Okwuashi O, Ndehedehe CE. Deep support vector machine for hyperspectral image classification. Pattern Recognition. 2020;103:107298-107298. Available from: https://dx.doi.org/10.1016/j.patcog.2020.107298.

30) Kalaiarasi G, Maheswari S. Deep proximal support vector machine classifiers for hyperspectral images classification. Neural Computing and Applications. 2021;33(20):13391-13415. Available from: https://dx.doi.org/10.1007/s00521-021-05965-0.

31) Wang L, Feng Y, Gao Y, Wang Z, He M. Compressed Sensing Reconstruction of Hyperspectral Images Based on Spectral Unmixing. IEEE Journal of Selected Topics in Applied Earth Observations and Remote Sensing. 2018;11(4):1266-1284. Available from: https://dx.doi.org/10.1109/jstars.2017.2787483.

32) Yang J, Qian J. Hyperspectral Image Classification via Multiscale Joint Collaborative Representation With Locally Adaptive Dictionary. IEEE Geoscience and Remote Sensing Letters. 2018;15(1):112-116. Available from: https://dx.doi.org/10.1109/lgrs.2017.2776113. 